\title{
The Extension of Integrable Mappings to Non-Commuting Variables
}

A. RAMANI ${ }^{1}$, T. TAMIZHMANI ${ }^{2}$, B. GRAMMATICOS ${ }^{3}$ and

K. M. TAMIZHMANI ${ }^{4}$

${ }^{1}$ CPT, Ecole Polytechnique, CNRS, UMR 7644, 91128 Palaiseau, France

E-mail: ramani@cpht.polytechnique.fr

${ }^{2}$ Dept. of Mathematics, Avvaiyar Government College for Women, Karaikal, India E-mail: arasi55@yahoo.com

${ }^{3}$ GMPIB, Université Paris VII Tour 24-14, 5 étage, case 7021, 75251 Paris, France E-mail: grammati@paris7.jussieu.fr

${ }^{4}$ Dept. of Mathematics, Pondicherry University, Kalapet, Pondicherry, 605014 India E-mail: tamizh@yahoo.com

This paper is part of the Proceedings of SIDE V;

Giens, June 21-26, 2002

\begin{abstract}
We present an extension of a family of second-order integrable mappings to the case where the variables do not commute. In every case we introduce a commutation rule which is consistent with the mapping evolution. Through the proper ordering of variables we ensure the existence of an invariant in the non-commuting case.
\end{abstract}

\section{Introduction}

Integrable equations involving non-commuting variables present a special interest, in particular in view of their applicability to quantum field theories. A large literature exists concerning supersymmetric or just fermionic extensions of integrable evolution equations. It is now clear that the special properties which characterise integrability can be extended to the case where the dependent variable involves fermionic as well as bosonic components.

In the domain of discrete systems, curiously, there has not been much progress in this direction. Few studies have been devoted to mappings involving non-commuting variables. In [1] the quantum integrability of a familly of (classically) integrable lattices has been related to the existence of a well-defined quantum Yang-Baxter structure, which provides a complete set of commuting operators. A more direct approach to quantum integrability has been used in [2] concerning a mapping of the QRT family. Starting from a restricted parametrization of the latter and postulating that the mapping variables $(x, y)$ are operators satisfying canonical relations $[x, y]=i \hbar$, it has been shown that it is 
possible to choose the right ordering so that the the classical invariant remains invariant even in the quantum case. In [3] we have presented an approach towards a combination of the two "quantisations", in the sense of Novikov [4], deautonomisation and real space quantisation. We have shown that the known Lax pairs of discrete Painlevé equations could be transformed, through the appropriate reordering, so as to obtain the corresponding discrete Painlevé equations from the compatibility conditions. Naturally in that work we have also considered the question of the quantisation of autonomous mappings.

In the present work we will return to the question of the proper quantisation of integrable second-order mappings. There are many reasons for this. First the number of cases treated in [3] is rather limited: it corresponded to the knowledge of discrete Painlevé equations at the beginning of the 90's which was, at best, fragmentary. Second, the treatment presented in [3] focused on mappings which are "symmetric" in the QRT terminology. However, for most cases, the natural form is an asymmetric one, so a way had to be found allowing the natural treatment of these cases. This, of course, necessitated the extension of the quantisation rules introduced in [3].

In what follows, we shall examine systematically the autonomous forms that were obtained from the classification of discrete Painlevé equations and presented in [5]. We shall introduce consistent quantification schemes and construct the corresponding invariants. The main difficulty for the quantisation of discrete systems is the fact that most commutation rules are incompatible with the evolutions induced by the mapping. By this we mean that if we assume that the mapping variables obey some commutation rules at some iteration, there is no guarantee whatsoever that at the next step the mapping variables will obey the same commutation rule. In particular, the Heisenberg commutation relation $[x, y]=1$ (and the associated Weyl procedure) is not necessarily the proper commutation rule for all the mappings we shall examine here (although it does work for a certain class). On the other hand there is no deep physical reason (such as the Hamiltonian structure in the case of the Heisenberg rule) why one should choose one rule over another. Thus we are free to explore exotic commutation rules. In [3] we have introduced the relation

$$
x y=q y x+\lambda(x+y)+\nu
$$

which is an extension of the Weyl commutation rule $x y=q y x$. However this rule is geared toward "symmetric" mappings where $x$ and $y$ play the same role. In what follows we shall allow for a genuine asymmetry between $x$ and $y$ and thus work with

$$
x y=q y x+\lambda x+\mu y+\nu
$$

In order to fix the notation in a more precise way we consider the iteration of the mapping to form the sequence:

$$
\ldots, y_{n-2}, x_{n-1}, y_{n}, x_{n+1}, y_{n+2}, \ldots
$$

i.e. the $x$ variables are associated to indices of one parity while the $y$ variables are related to indices of the other parity. In that way we distinguish clearly the noncommuting variables and work explicitly in a non "symmetric" frame. In the light of this notation the commutation rules (1.2) is to be understood between $y_{n}$ and $x_{n-1}$ (or $x_{n+1}$ ). 


\section{Non-commuting forms of QRT mappings}

In what follows, we shall examine mappings which in the usual, commuting case, possess an invariant of the form $K(X, Y)$. By this we mean that the equation $K\left(X, y_{n}\right)=K\left(x_{n-1}, y_{n}\right)$ is satisfied, identically for $X=x_{n-1}$ and also for $X=x_{n+1}$ where $x_{n+1}$ is related to $y_{n}$, $x_{n-1}$ through the evolution of the mapping. (Similarly $K\left(x_{n+1}, Y\right)=K\left(x_{n+1}, y_{n}\right)$ is satisfied by both $Y=y_{n}$ and $\left.Y=y_{n+2}\right)$. These are the properties we shall ensure in the non-commuting case, through the proper choice of commutation rules, invariant and mapping.

The family of mappings we are going to work with is the one introduced in [6] and which came to be known as the QRT mapping. Their general expression in the commuting case is:

$$
\begin{gathered}
x_{n+1}=\frac{f_{1}\left(y_{n}\right)-x_{n-1} f_{2}\left(y_{n}\right)}{f_{2}\left(y_{n}\right)-x_{n-1} f_{3}\left(y_{n}\right)} \\
y_{n+2}=\frac{g_{1}\left(x_{n+1}\right)-y_{n} g_{2}\left(x_{n+1}\right)}{g_{2}\left(x_{n+1}\right)-y_{n} g_{3}\left(x_{n+1}\right)}
\end{gathered}
$$

where $f_{i}, g_{i}$ are specific polynomials of degree not exceeding four, involving at maximum 8 nontrivial parameters. The invariant of the mapping (2.1) can be written as a rational expression $K(X, Y)$ the numerator and denominator of which are biquadratic in $X$ and $Y$ and invariance has to be understood as in the paragraph above.

The classification of the canonical forms of (2.1) was presented in [7]. It follows, in some sense, the more general classification obtained [5] in the case of discrete Painlevé equations (which are the nonautonomous extensions of the QRT mapping), based on affine Weyl groups. However, in the autonomous case we shall consider here, the classification of [7] is sufficient.

I) We start with the mapping:

$$
x_{n+1}+x_{n-1}=F\left(y_{n}\right)
$$

The commutation relation we are going to use in this case is

$$
y_{n} x_{n-1}=x_{n-1} y_{n}+k
$$

where a priori $k$ may be a function of $y_{n}$ only. Using (2.2) it is straightforward to check that $y_{n} x_{n+1}+y_{n} x_{n-1}=x_{n+1} y_{n}+x_{n-1} y_{n}$ or

$$
x_{n+1} y_{n}=y_{n} x_{n+1}+k
$$

Guided by the commutative case and the results of [2], we introduce the invariant:

$$
K(X, Y)=X^{2} f(Y)+f(Y) X^{2}+X g(Y)+g(Y) X+2 h(Y)
$$

and require that the equation $K\left(X, y_{n}\right)=K\left(x_{n-1}, y_{n}\right)$ be satisfied not only by $X=x_{n-1}$ but also for $X=x_{n+1}$ for $x_{n+1}$ defined by (2.2) for some $F\left(y_{n}\right)$ (which is to be related to $f, g$ and $h)$. We use the notation $K=K\left(x_{n-1}, y_{n}\right)$ and $\hat{K}=K\left(x_{n+1}, y_{n}\right)$. We have

$$
K=x_{n-1}\left(x_{n-1} f+g\right)+\left(f x_{n-1}+g\right) x_{n-1}+2 h
$$




$$
\hat{K}=x_{n+1}\left(x_{n+1} f+g\right)+\left(f x_{n+1}+g\right) x_{n+1}+2 h
$$

Let us show that if $F=-g f^{-1}$, i.e. $x_{n+1}=-x_{n-1}-g f^{-1}$ then we indeed have the conservation $\hat{K}=K$. The essential ingredient we need in the proof is the commutation rule between $x_{n \pm 1}$ and a function of $y_{n}$. Using (2.3-4) and the fact that $k$ commutes with $y_{n}$ (this is of course crucial) one can easily show that for any function $j\left(y_{n}\right)$

$$
j\left(y_{n}\right) x_{n \pm 1}=x_{n \pm 1} j\left(y_{n}\right) \mp k j^{\prime}\left(y_{n}\right)
$$

where the prime denotes the derivation with respect to the argument. So

$$
\begin{gathered}
K-2 h=x_{n-1}\left(-x_{n+1} f\right)+\left(-f x_{n+1}\right) x_{n-1}= \\
=x_{n-1}\left(-f x_{n+1}-k f^{\prime}\right)+\left(-x_{n+1} f+k f^{\prime}\right) x_{n-1} \\
=-x_{n-1} f x_{n+1}-x_{n+1} f x_{n-1}+k f^{\prime} x_{n-1}-x_{n-1} k f^{\prime}= \\
=-x_{n-1} f x_{n+1}-x_{n+1} f x_{n-1}+k\left(k f^{\prime}\right)^{\prime}
\end{gathered}
$$

Following the same steps we find that:

$$
\hat{K}-2 h=x_{n+1}\left(-x_{n-1} f\right)+\left(-f x_{n-1}\right) x_{n+1}=-x_{n+1} f x_{n-1}-x_{n-1} f x_{n+1}+k\left(k f^{\prime}\right)^{\prime}
$$

So the quantities $K$ and $\hat{K}$ indeed coincide. Note that one should be very careful when commuting $k$ with the $x$ variables since it is not assumed that $k$ is a $c$-number, but only that it is a function of $y_{n}$. Thus the mapping (2.2) possesses the invariant (2.5) in the sense explained at the beginning of this section, provided $F=-g f^{-1}$.

We must now complement (2.2) with an equation relating $y_{n}, x_{n+1}$ and $y_{n+2}$. The simplest choice would be to write an equation of the same form, namely

$$
y_{n+2}+y_{n}=G\left(x_{n+1}\right)
$$

Following the same reasoning as above we see that this will be compatible with the commutation rule (2.4) if $k$ commutes with $x_{n+1}$. But in this case $k$ must indeed be a $c$-number, since it must already commute with $y_{n}$. Then using (2.4) and (2.8) we find

$$
y_{n+2} x_{n+1}=x_{n+1} y_{n+2}+k
$$

So equation (2.3) will indeed be valid for all $n$ and thus also (2.4). Moreover we need $\hat{K}$ to be of the form

$$
\hat{K}=\phi\left(x_{n+1}\right) y_{n+2}^{2}+y_{n+2}^{2} \phi\left(x_{n+1}\right)+\psi\left(x_{n+1}\right) y_{n+2}+y_{n+2} \psi\left(x_{n+1}\right)+2 \omega\left(x_{n+1}\right)
$$

We can retrace the steps of the above reasoning and show that if $G=-\psi \phi^{-1}$ then $K\left(x_{n+1}, y_{n+2}\right)=K\left(x_{n+1}, y_{n}\right)$. Equation (2.10) is indeed a rewriting of (2.5) provided $f$, $g$ and $h$ are quadratic in $y_{n}$ and then $\phi, \psi$ and $\omega$ are also quadratic in $x_{n+1}$. In fact $K$ can be explicitly written:

$$
\begin{aligned}
K(X, Y)=\alpha\left(X^{2} Y^{2}\right. & \left.+Y^{2} X^{2}\right)+\beta\left(X^{2} Y+Y X^{2}\right)+2 \gamma X^{2} \\
& +\delta\left(X Y^{2}+Y^{2} X\right)+\epsilon(X Y+Y X)+2 \zeta X+2 \kappa Y^{2}+2 \lambda Y+2 \mu
\end{aligned}
$$


This result was already obtained in [2]. Note that if $\delta=\beta, \kappa=\gamma$ and $\lambda=\zeta$ then $K$ is in fact symmetric in $X$ and $Y$. Then as functions of their argument, $\phi=f, \psi=g, \omega=h$ and $G=F$. This is the so-called "symmetric" QRT case and there would be no point giving different names $x$ and $y$ to odd- and even-numbered variables. The commutation rule (2.4) is just (2.3) written at $n+1$ and similarly (2.8) is just (2.2) at $n+1$.

II) Next we examine the mapping:

$$
y_{n+2} y_{n}=G\left(x_{n+1}\right)
$$

This mapping is compatible with more than one instance of the rule (1.1). Indeed, both:

$$
x_{n+1} y_{n}=y_{n} x_{n+1}+\nu y_{n}
$$

and

$$
x_{n+1} y_{n}=q y_{n} x_{n+1}
$$

lead to consistent rules for the commutation of $y_{n+2}$ with $x_{n+1}$. Indeed combining (2.12) with (2.13) we get

$$
y_{n+2} x_{n+1}=x_{n+1} y_{n+2}+\nu y_{n+2}
$$

while (2.12) and (2.14) lead to

$$
y_{n+2} x_{n+1}=q x_{n+1} y_{n+2}
$$

Both cases can be represented by the shorthand

$$
x_{n+1} y_{n}=y_{n} \tilde{x}_{n+1}, \quad y_{n+2} x_{n+1}=\tilde{x}_{n+1} y_{n+2}
$$

with $\tilde{x}_{n+1}$ being $x_{n+1}+\nu$ in the first case and $q x_{n+1}$ in the second one. In both cases $\tilde{x}_{n+1}$ is just a function of $x_{n+1}$ and commutes with it.

Next we introduce the invariant (obtained from the commutative case of QRT mapping with the appropriate ordering):

$$
K(X, Y)=Y f(X)+f(X) Y+2 g(X)+Y^{-1} h(X)+h(X) Y^{-1}
$$

This assumes of course that $Y$ is invertible. We have so far never specified what space of noncommuting objects we are dealing with, whether they are operators, whether divisors of zero exist, and so on. At this point we assume that the generic object is invertible and we are not going to worry about the possibility of $Y$ reaching a singular, noninvertible value (not any more than the exceptional occurence of a zero in the commutative case). We shall show that the conservation law $K\left(x_{n+1}, Y\right)=K\left(x_{n+1}, y_{n}\right)$ is satisfied, identically by $Y=y_{n}$, but also by $Y=y_{n+2}$ for $y_{n+2}$ satisfying (2.12) for the appropriate value of $G$ expressed in terms of $f, g$ and $h$. In order to prove this we shall use the following properties, which are consequences of (2.17):

$$
\begin{gathered}
j\left(x_{n+1}\right) y_{n}=y_{n} j\left(\tilde{x}_{n+1}\right), \quad y_{n+2} j\left(x_{n+1}\right)=j\left(\tilde{x}_{n+1}\right) y_{n+2} \\
j\left(\tilde{x}_{n+1}\right) y_{n}^{-1}=y_{n}^{-1} j\left(x_{n+1}\right), \quad y_{n+2}^{-1} j\left(\tilde{x}_{n+1}\right)=j\left(x_{n+1}\right) y_{n+2}^{-1}
\end{gathered}
$$

for any function $j$. Using (2.19) we can rewrite $K\left(x_{n+1}, y_{n}\right)$ as

$$
K\left(x_{n+1}, y_{n}\right)=y_{n}\left(f\left(x_{n+1}\right)+f\left(\tilde{x}_{n+1}\right)\right)+2 g\left(x_{n+1}\right)+\left(h\left(\tilde{x}_{n+1}\right)+h\left(x_{n+1}\right)\right) y_{n}^{-1}
$$


while $K\left(x_{n+1}, y_{n+2}\right)$ can be rewritten as:

$$
K\left(x_{n+1}, y_{n+2}\right)=\left(f\left(\tilde{x}_{n+1}\right)+f\left(x_{n+1}\right)\right) y_{n+2}+2 g\left(x_{n+1}\right)+y_{n+2}^{-1}\left(h\left(x_{n+1}\right)+h\left(\tilde{x}_{n+1}\right)\right)
$$

These two quantities will coincide if (2.12) is satisfied with

$$
G\left(x_{n+1}\right)=\left(h\left(\tilde{x}_{n+1}\right)+h\left(x_{n+1}\right)\right)\left(f\left(x_{n+1}\right)+f\left(\tilde{x}_{n+1}\right)\right)^{-1}
$$

Up to this point the precise form of $f, g$ and $h$ (and thus of $G$ ) has not been fixed. The choice will depend on the equation for $x_{n-1}, y_{n}$ and $x_{n+1}$ that we wish to use. The simplest case would be to consider an equation similar to (2.12)

$$
x_{n+1} x_{n-1}=F\left(y_{n}\right)
$$

The commutation rule (2.13) is not compatible with this choice but we can use (2.14) and then from (2.14) and (2.23) we deduce

$$
y_{n} x_{n-1}=q x_{n-1} y_{n}
$$

which means that (2.16) (and thus also (2.14)) will be satisfied for all $n$. We can reproduce the above steps provided $K$ of (2.18) can be rewritten as:

$$
K(X, Y)=X \phi(Y)+\phi(Y) X+2 \psi(Y)+X^{-1} \omega(Y)+\omega(Y) X^{-1}
$$

This means that $f, g$ and $h$ are linear in $X$ and $X^{-1}$ and thus $\phi, \psi$ and $\omega$ are similarly linear in $Y$ and $Y^{-1}$. In fact we have:

$$
\begin{aligned}
K(X, Y)= & \alpha(X Y+Y X)+2 \beta X+\gamma\left(X Y^{-1}+Y^{-1} X\right)+2 \delta Y \\
& +2 \epsilon+2 \zeta Y^{-1}+\kappa\left(X^{-1} Y+Y X^{-1}\right)+2 \lambda X^{-1}+\mu\left(X^{-1} Y^{-1}+Y^{-1} X^{-1}\right)
\end{aligned}
$$

Again, if $\delta=\beta, \kappa=\gamma$ and $\lambda=\zeta, K$ is symmetric in $X$ and $Y$. We have $\phi=f, \psi=g$, $\omega=h, G=F$ and odd- and even-numbered variables should really have the same name.

Another possibility, however, does exist. The commutation rule (2.13) is of the form (2.4) with the choice $k\left(y_{n}\right)=\nu y_{n}$. We saw above that (2.4) is compatible with $(2.2)$ provided the commutation rule (2.3) holds. But again, (2.13) implies (2.15) if (2.12) holds, and note that (2.15) is nothing but (2.3) written at $n+2$. So it follows that one can use commutation rules (2.3) and (2.4) for all $n$ with the proper choice $k\left(y_{n}\right)=\nu y_{n}$, and this will be compatible with the coupling of $(2.2)$

$$
x_{n+1}+x_{n-1}=F\left(y_{n}\right)
$$

with (2.12)

$$
y_{n+2} y_{n}=G\left(x_{n+1}\right) .
$$

In this case the situation is strongly asymmetric between the $x$ 's and the $y$ 's, the former satisfying an additive equation, the latter a multiplicative one [8]. Moreover the commutation rule treats the variables in a different way. The constant $K(X, Y)$ must be of the form (2.5) and also of the form (2.18). This is realised by:

$$
K(X, Y)=\alpha\left(X^{2} Y+Y X^{2}\right)+2 \beta X^{2}+\gamma\left(X^{2} Y^{-1}+Y^{-1} X^{2}\right)
$$




$$
+\delta(X Y+Y X)+2 \epsilon X+\zeta\left(X Y^{-1}+Y^{-1} X\right)+2 \kappa Y+2 \lambda+2 \mu Y^{-1}
$$

The invariance of $K$ under the evolution is a consequence, separately, of the arguments we used in subsections I and II with the appropriate choices for $F$ in (2.2) and $G$ in (2.12) in terms of the parameters of (2.27) and $\nu$.

III) In this subsection we shall we examine mappings of the form:

$$
\left(x_{n+1}+y_{n}\right)\left(x_{n-1}+y_{n}\right)=F\left(y_{n}\right)
$$

The commutation rule we shall introduce here is

$$
y_{n} x_{n-1}=x_{n-1} y_{n}+\nu\left(x_{n-1}+y_{n}\right)
$$

Starting from this relation and using the mapping (2.28) we can show that

$$
x_{n+1} y_{n}=y_{n} x_{n+1}+\nu\left(x_{n+1}+y_{n}\right)
$$

Assuming that at even steps we have the same form as (2.28)

$$
\left(x_{n+1}+y_{n+2}\right)\left(x_{n+1}+y_{n}\right)=G\left(x_{n+1}\right)
$$

the commutation rules (2.29) and (2.30) will hold for all $n$ 's. We can show that (2.28-31) are indeed consistent with some invariant, for appropriate values of $F$ and $G$. We start from:

$$
\begin{gathered}
K(X, Y)=X^{2}(X+Y)^{-1} f(Y)+f(Y)(X+Y)^{-1} X^{2}+X(X+Y)^{-1} g(Y)+ \\
g(Y)(X+Y)^{-1} X+(X+Y)^{-1} h(Y)+h(Y)(X+Y)^{-1}
\end{gathered}
$$

In order to show the invariance of (2.32) under the evolution, which means $K\left(x_{n+1}, y_{n}\right)=$ $K\left(x_{n-1}, y_{n}\right)$ (and $\left.K\left(x_{n+1}, y_{n+2}\right)=K\left(x_{n+1}, y_{n}\right)\right)$ we have to write $K\left(x_{n-1}, y_{n}\right)$ as

$$
K\left(x_{n-1}, y_{n}\right)=\left(x_{n-1}+y_{n}\right) P\left(y_{n}\right)+Q\left(y_{n}\right)+R\left(y_{n}\right)\left(x_{n-1}+y_{n}\right)^{-1}
$$

The method is elementary. Wherever we have $x_{n-1}$ in $K$ we write it as $w_{n}-y_{n}$ (i.e. $\left.w_{n}=x_{n-1}+y_{n}\right)$ so everything can be written in terms of $w_{n}$ and $y_{n}$. Now the commutation rule of $y_{n}$ with $w_{n}$ is quite simple. From (2.29) it follows that:

$$
y_{n} w_{n}=w_{n} y_{n}+\nu w_{n}=w_{n} \tilde{y}_{n}
$$

where $\tilde{y}_{n} \equiv y_{n}+\nu$. So it follows that for any function $j$ :

$$
j\left(y_{n}\right) w_{n}=w_{n} j\left(\tilde{y}_{n}\right)
$$

This allows us to move functions of $y_{n}$ left and right of $w_{n}$ (or $w_{n}^{-1}$ ) with just redefinition of the arguments of the functions. The terms involving $h$ lead only to $w_{n}^{-1}$, those involving $g$ lead to $w_{n}^{-1}$ terms and $w_{n}$-independent terms (coming, respectively from $-y_{n}$ and $w_{n}$ in the expansion of $x_{n}$ ), while the $f$ terms contribute $w_{n}$ terms in addition to $w_{n}^{-1}$ terms and $w_{n}$-independent terms. Because of $(2.35)$ we are always allowed to bring functions of $y_{n}$ to the side we prefer, at the price of redefining the arguments, so we can indeed 
write $K\left(x_{n-1}, y_{n}\right)$ as (2.33). Consider now the commutation rule (2.30) of $y_{n}$ with $x_{n+1}$. Defining $v_{n}=x_{n+1}+y_{n}$ we see that

$$
v_{n} y_{n}=y_{n} v_{n}+\nu v_{n}=\tilde{y}_{n} v_{n}
$$

For any function $j$ we have

$$
v_{n} j\left(y_{n}\right)=j\left(\tilde{y}_{n}\right) v_{n}
$$

so commuting $v_{n}$ from left to right of a function of $y_{n}$ has the same effect as commuting $w_{n}$ from right to left. So it follows that if we start from $K\left(x_{n+1}, y_{n}\right)$, write $x_{n+1}=v_{n}-y_{n}$ and do exactly the same manipulations but in the opposite direction we will find

$$
K\left(x_{n+1}, y_{n}\right)=P\left(y_{n}\right)\left(x_{n+1}+y_{n}\right)+Q\left(y_{n}\right)+\left(x_{n+1}+y_{n}\right)^{-1} R\left(y_{n}\right)
$$

where $P, Q$ and $R$ are expressed in terms $y_{n}, \tilde{y}_{n}$ and of $f, g, h$ at various arguments $\left(y_{n}, \tilde{y}_{n}\right.$, etc.) but these expressions, however complicated, will be exactly the same as those in (2.33). It follows that if we choose $\left(x_{n+1}+y_{n}\right)=P\left(y_{n}\right)^{-1} R\left(y_{n}\right)\left(x_{n-1}+y_{n}\right)^{-1}$ the first term in the r.h.s of (2.38) coincides with the last one in (2.33) and conversely, so $K\left(x_{n+1}, y_{n}\right)$ and $K\left(x_{n-1}, y_{n}\right)$ indeed coincide. So with the choice $F\left(y_{n}\right)=P\left(y_{n}\right)^{-1} R\left(y_{n}\right)$, the mapping (2.28) leaves $K$ invariant. In order to be able to do the same manipulation at even steps, we must be able to rewrite $K\left(x_{n+1}, y_{n}\right)$ in similar form as $(2.32)$ in terms of $y_{n}^{2}, y_{n}$ and functions of $x_{n+1}$. For this it is necessary and sufficient that $f, g$ and $h$ be functions of degree in $y_{n}$ not higher than two. The very same steps lead to equation (2.31). The expression of $K$ must be

$$
\begin{gathered}
K(X, Y)=\alpha\left(X^{2} W^{-1} Y^{2}+Y^{2} W^{-1} X^{2}\right)+\beta\left(X^{2} W^{-1} Y+Y W^{-1} X^{2}\right) \\
+\gamma\left(X^{2} W^{-1}+W^{-1} X^{2}\right)+\delta\left(X W^{-1} Y^{2}+Y^{2} W^{-1} X\right)+\epsilon\left(X W^{-1} Y+Y W^{-1} X\right) \\
+\zeta\left(X W^{-1}+W^{-1} X\right)+\kappa\left(W^{-1} Y^{2}+Y^{2} W^{-1}\right)+\lambda \\
\left(W^{-1} Y+Y W^{-1}\right)+2 \mu W^{-1}
\end{gathered}
$$

with $W=X+Y$. As previously, the choices $\delta=\beta, \kappa=\gamma$ and $\lambda=\zeta$ lead to $K$ symmetric in $X$ and $Y$ and variables with even and odd indices should have the same name.

IV) Here we consider the mapping

$$
\left(x_{n+1} y_{n}-1\right)\left(y_{n} x_{n-1}-1\right)=F\left(y_{n}\right)
$$

and we will not keep any suspense but give directly the equation at even n's:

$$
\left(y_{n+2} x_{n+1}-1\right)\left(x_{n+1} y_{n}-1\right)=G\left(x_{n+1}\right)
$$

These two equations are compatible with the commutation rule

$$
y_{n} x_{n-1}=q x_{n-1} y_{n}+(1-q)
$$

i.e.

$$
y_{n} x_{n-1}-1=q\left(x_{n-1} y_{n}-1\right)
$$


Calling $w_{n}$ the quantity $\left(y_{n} x_{n-1}-1\right)$ one has $w_{n} y_{n}=q y_{n} w_{n}$. Using this relation in combination with (2.40) it is easy to show that $y_{n} v_{n}=q v_{n} y_{n}$, where $v_{n}=\left(x_{n+1} y_{n}-1\right)$ and thus

$$
x_{n+1} y_{n}-1=q\left(y_{n} x_{n+1}-1\right)
$$

Using the latter with (2.42) one shows that (2.43) is satisfied at all $n$ 's and thus so is (2.44). The invariant will be:

$$
\begin{gathered}
K(X, Y)=X^{2}(Y X-1)^{-1} f(Y)+f(Y)(X Y-1)^{-1} X^{2} \\
+X(Y X-1)^{-1} g(Y)+g(Y)(X Y-1)^{-1} X+ \\
(Y X-1)^{-1} h(Y)+h(Y)(X Y-1)^{-1}
\end{gathered}
$$

We start from $K\left(x_{n-1}, y_{n}\right)$ and rewrite it into a more convenient form. When $x_{n-1}$ is on the left of $w_{n}^{-1} \equiv\left(y_{n} x_{n-1}-1\right)^{-1}$ we write it as $y_{n}\left(w_{n}+1\right)^{-1}$, while when it is on the right of $\left(x_{n-1} y_{n}-1\right)^{-1}$ we use the form $\left(x_{n-1} y_{n}-1+1\right) y_{n}^{-1}$ which thanks to $(2.43)$ can also be written in terms of $w_{n}$. Because one has $w_{n} j\left(y_{n}\right)=j\left(q y_{n}\right) w_{n}$ for any function $j$, one can move $w_{n}$ at will to the left or to the right of functions of $y_{n}$ by taking proper care of the arguments. Just as in the previous case, the $h$ terms give $w_{n}^{-1}$ terms, the $g$ terms give these and also $w_{n}$-independent terms while the $f$ terms lead, in addition, to terms proportional to $w_{n}$. We may move the $w_{n}$ 's to the side we want and thus write $K\left(x_{n-1}, y_{n}\right)$ as:

$$
K\left(x_{n-1}, y_{n}\right)=w_{n} P\left(y_{n}\right)+Q\left(y_{n}\right)+R\left(y_{n}\right) w_{n}^{-1}
$$

Now, because of (2.44), any operation we do with $x_{n+1}$ (and $v_{n}$ ) going from left to right, say, will give exactly the same terms as those coming from $x_{n-1}$ (and $w_{n}$ ) going from right to left. However complicated $P, Q$ and $R$ are in terms of $f, g$ and $h$ we will recover exactly

$$
K\left(x_{n+1}, y_{n}\right)=P\left(y_{n}\right) v_{n}+Q\left(y_{n}\right)+v_{n}^{-1} R\left(y_{n}\right)
$$

So $K$ will indeed be an invariant if $v_{n}=P\left(y_{n}\right)^{-1} R\left(y_{n}\right) w_{n}^{-1}$ which is exactly (2.40) with $F=P^{-1} Q$.

In order to find (2.41), we need to be able to rewrite $K\left(x_{n+1}, y_{n}\right)$ in a form similar to (2.45) in terms of $y_{n}^{2}, y_{n}$ and functions of $x_{n+1}$. This can be realised if $f, g$ and $h$ are also quadratic in $y_{n}$. The expression of $K$ is quite similar to (2.39) but one must remember that instead of a single $W^{-1}$ the object in "sandwich" between $X^{a}$ and $Y^{b}$ is $(Y X-1)^{-1}$ while it becomes $(X Y-1)^{-1}$ between $Y^{b}$ and $X^{a}$ (even if $a$ and/or $b$ vanish, so the last term is $\left.\mu\left((Y X-1)^{-1}+(X Y-1)^{-1}\right)\right)$. Again a symmetric case is obtained if $\delta=\beta, \kappa=\gamma$ and $\lambda=\zeta$.

\section{Non-commuting forms of mappings equivalent to QRT}

So far we have considered QRT forms (2.1) in which the l.h.s. was given in polynomial form. Here we shall extend our treatment to the case where the l.h.s. of the mapping is rational [9].

I) Consider the mapping

$$
\frac{\left(x_{n+1}+y_{n}+Z\right)\left(x_{n-1}+y_{n}+Z\right)}{\left(x_{n+1}+y_{n}\right)\left(x_{n-1}+y_{n}\right)}=F\left(y_{n}\right)
$$




$$
\frac{\left(x_{n+1}+y_{n+2}+Z\right)\left(x_{n+1}+y_{n}+Z\right)}{\left(x_{n+1}+y_{n+2}\right)\left(x_{n+1}+y_{n}\right)}=G\left(x_{n+1}\right)
$$

for commuting variables, where $Z$ is a constant. There are different ways to write it if the variables do not commute. We choose:

$$
\begin{gathered}
\left(x_{n+1}+y_{n}+Z\right)\left(x_{n+1}+y_{n}\right)^{-1}\left(x_{n-1}+y_{n}+Z\right)\left(x_{n-1}+y_{n}\right)^{-1}=F\left(y_{n}\right) \\
\left(x_{n+1}+y_{n+2}+Z\right)\left(x_{n+1}+y_{n+2}\right)^{-1}\left(x_{n+1}+y_{n}+Z\right)\left(x_{n+1}+y_{n}\right)^{-1}=G\left(x_{n+1}\right)
\end{gathered}
$$

It is not easy at first sight to find a consistent commutation rule, unless one notices that one can rewrite (3.1) in terms of four variables by introducing $w_{n}=\left(x_{n-1}+y_{n}+Z\right)\left(x_{n-1}+y_{n}\right)^{-1}$ and $v_{n+1}=\left(x_{n+1}+y_{n}+Z\right)\left(x_{n+1}+y_{n}\right)^{-1}$. We find that (3.2) and the definitions of $w_{n}$ and $v_{n+1}$ give the system:

$$
\begin{gathered}
y_{n}+x_{n-1}=Z\left(w_{n}-1\right)^{-1} \\
v_{n+1} w_{n}=F\left(y_{n}\right) \\
x_{n+1}+y_{n}=Z\left(v_{n+1}-1\right)^{-1} \\
w_{n+2} v_{n+1}=G\left(x_{n+1}\right)
\end{gathered}
$$

The sequence of variables is now

$$
\ldots, y_{n-2}, v_{n-1}, x_{n-1}, w_{n}, y_{n}, v_{n+1}, x_{n+1}, w_{n+2}, y_{n+2}, \ldots
$$

Because of the form of the left-hand sides one can find consistent commutation rules between any two consecutive variables of the above sequence. Since we have an alternance of additive and multiplicative equations, we look at a rule similar to (2.13) rather than (2.14). More precisely we take

$$
y_{n} w_{n}=w_{n} y_{n}+\nu w_{n} \equiv w_{n} \tilde{y}_{n}
$$

which implies, on the one hand

$$
w_{n} x_{n-1}=x_{n-1} w_{n}+\nu w_{n}
$$

because of (3.3a), and also

$$
v_{n+1} y_{n}=\tilde{y}_{n} v_{n+1}=y_{n} v_{n+1}+\nu v_{n+1}
$$

because of (3.3b). Using (3.3c) we further find $x_{n+1} v_{n+1}=v_{n+1} \tilde{x}_{n+1}$ where $\tilde{x}_{n+1}=$ $x_{n+1}+\nu$, and finally (3.3d) leads to $w_{n+2} x_{n+1}=\tilde{x}_{n+1} w_{n+2}$ which is just (3.6) written two units further. This means that all these commutation rules are valid for all $n$ 's. There is no simple relation expressing directly the commutation of $x_{n-1}$, say, and $y_{n}$. On the other hand, since separately equations (3.3) are not of QRT type, we do not expect invariants involving two consecutive variables in (3.4). Invariance must still be understood, as throughout the paper, as under the incrementation of the index of either $x$ or $y$ by two units. It turns out, however, that using usual QRT invariants $K(X, Y)$ with the appropriate ordering, but using the intermediate variables to write them in an appropriate way, we can show the invariance under (3.2). Namely, inspired by the commutative case, we define:

$$
K(X, Y)=X^{2} U^{-1} f(Y)+f(Y) U^{-1} X^{2}+X U^{-1} g(Y)+
$$




$$
g(Y) U^{-1} X+U^{-1} h(Y)+h(Y) U^{-1}
$$

with $U=(X+Y+Z)(X+Y)$. Note that, whatever the commutations of $X$ and $Y$ are, $X+Y$ commutes with any function of itself. It follows that one can write:

$$
\begin{gathered}
U^{-1}=Z^{-1}\left((X+Y)^{-1}-(X+Y+Z)^{-1}\right)= \\
Z^{-2}\left((X+Y+Z)(X+Y)^{-1}-(X+Y)(X+Y+Z)^{-1}\right)
\end{gathered}
$$

The last term in (2.45) shows that the terms coming from $h$ in $K\left(x_{n-1}, y_{n}\right)$ (resp. in $K\left(x_{n+1}, y_{n}\right)$ ) can be written in terms of only $y_{n}$ and $w_{n}$ (resp. only $y_{n}$ and $v_{n+1}$ ). Moreover using the middle expression in (2.45) we can easily show that it is also true for the $g$ terms. Indeed

$$
\begin{gathered}
X U^{-1}=Z^{-1}\left((X+Y-Y)(X+Y)^{-1}-(X+Y+Z-Y-Z)(X+Y+Z)^{-1}\right) \\
=Z^{-1}\left((Y+Z)(X+Y+Z)^{-1}-Y(X+Y)^{-1}\right) \\
=Z^{-2}\left((Y+Z)\left(1-(X+Y)(X+Y+Z)^{-1}\right)-Y\left((X+Y+Z)(X+Y)^{-1}-1\right)\right)
\end{gathered}
$$

Only $y$ 's and $w$ or $v$ enter in these expression and the same is true, with a different ordering, for $U^{-1} X$. We will now proceed to show that that this is still the case for $X^{2} U^{-1}$ (and, similarly for $U^{-1} X^{2}$ ). To compute $X^{2} U^{-1}$ we first note that in the last term of (3.9) it is possible to move the functions of $Y$ to the right of the relevant object which are $w_{n}, w_{n}^{-1}$ or $v_{n+1}, v_{n+1}^{-1}$ depending on whether we work on $K\left(x_{n-1}, y_{n}\right)$ or $K\left(x_{n+1}, y_{n}\right)$. Because of (3.5) and (3.7) we have

$$
\begin{gathered}
X U^{-1}=Z^{-2}\left(Y+Z-(X+Y)(X+Y+Z)^{-1}(Y+Z \pm \nu)-\right. \\
\left.\left.(X+Y+Z)(X+Y)^{-1}(Y \mp \nu)+Y\right)\right)
\end{gathered}
$$

with the upper sign for $x_{n+1}$ and the lower one for $x_{n-1}$ But this can be rewritten as

$$
\begin{gathered}
X U^{-1}=Z^{-2}\left(\left(1-(X+Y)(X+Y+Z)^{-1}\right)(Y+Z \pm \nu)-\right. \\
\left.\left.\quad\left((X+Y+Z)(X+Y)^{-1}-1\right)(Y \mp \nu)\right)\right) \\
=Z^{-1}\left((X+Y+Z)^{-1}(Y+Z \pm \nu)-(X+Y)^{-1}(Y \mp \nu)\right)
\end{gathered}
$$

The functions of $Y$ on the right do not pose any problem, they just combine with $f$. The crucial point is that now, in computing $X^{2} U^{-1}$, we need only to compute $X(X+Y)^{-1}$ and $X(X+Y+Z)^{-1}$. So the same manipulations as in (3.9) will allow to write these quantities in terms of $w_{n}, w_{n}^{-1}$ and functions of $y_{n}$ only (resp. $v_{n+1}, v_{n+1}^{-1}$ and functions of $y_{n}$ only). Since everything will be similar for $U^{-1} X^{2}$, we see that all the terms in $K$ can be written in that form. Then using the commutation rules it is possible to write $K\left(x_{n-1}, y_{n}\right)$ as:

$$
K\left(x_{n-1}, y_{n}\right)=w_{n} P\left(y_{n}\right)+Q\left(y_{n}\right)+R\left(y_{n}\right) w_{n}^{-1}
$$

where $P, Q$ and $R$ are very complicated functions of $f, g$ and $h$. But comparing (3.5) and (3.7) we see that everything done with $v_{n+1}$, moving left to right has precisely the same effect as moving $w_{n}$ from right to left and conversely. So starting from $K\left(x_{n+1}, y_{n}\right)$ and redoing the same manipulations, but in the opposite direction we find that:

$$
K\left(x_{n+1}, y_{n}\right)=P\left(y_{n}\right) v_{n+1}+Q\left(y_{n}\right)+v_{n+1}^{-1} R\left(y_{n}\right)
$$


with the very same functions $P, Q$ and $R$. So if $v_{n+1} w_{n}=P\left(y_{n}\right)^{-1} R\left(y_{n}\right),(3.12)$ and (3.13) show that $K$ is conserved. This is precisely equation (3.2a) with $F=P^{-1} R$. In order to find $(3.2 \mathrm{~b})$ we must be able to rewrite $K\left(x_{n+1}, y_{n}\right)$ as an expression quadratic in $y_{n}$. This means that $f, g$ and $h$ are quadratic and the form of $K$ is just the same as (2.39) with $U=(X+Y+Z)(X+Y)$ replacing $W$.

II) We now turn to the the mapping

$$
\begin{gathered}
\left(x_{n+1} y_{n}-Z\right)\left(x_{n+1} y_{n}-1\right)^{-1}\left(y_{n} x_{n-1}-Z\right)\left(y_{n} x_{n-1}-1\right)^{-1}=F\left(y_{n}\right) \\
\left(y_{n+2} x_{n+1}-Z\right)\left(y_{n+2} x_{n+1}-1\right)^{-1}\left(x_{n+1} y_{n}-Z\right)\left(x_{n+1} y_{n}-1\right)^{-1}=G\left(x_{n+1}\right)
\end{gathered}
$$

We use the same sequence of variables (3.4), and (3.14ab) are just as (3.3a,c) but here $w_{n}$ and $v_{n+1}$ are determined by

$$
\begin{gathered}
w_{n}=\left(y_{n} x_{n-1}-Z\right)\left(y_{n} x_{n-1}-1\right)^{-1} \\
v_{n+1}=\left(x_{n+1} y_{n}-Z\right)\left(x_{n+1} y_{n}-1\right)^{-1}
\end{gathered}
$$

So

$$
\begin{gathered}
y_{n} x_{n-1}=1+(1-Z)\left(w_{n}-1\right)^{-1} \\
x_{n+1} y_{n}=1+(1-Z)\left(v_{n+1}-1\right)^{-1}
\end{gathered}
$$

Since all four equations of the system at hand, namely (3.16a), (3.3b), (3.16b), (3.3d) are multiplicative, the commutation rules we have to use are not (3.5-7) but

$$
\begin{aligned}
w_{n} x_{n-1} & =q x_{n-1} w_{n} \\
y_{n} w_{n} & =q w_{n} y_{n} \\
v_{n+1} y_{n} & =q y_{n} v_{n+1} \\
x_{n+1} v_{n+1} & =q v_{n+1} x_{n+1}
\end{aligned}
$$

Then (3.3d) leads to $w_{n+2} x_{n+1}=q x_{n+1} w_{n+2}$, which is just (3.17a) written two units further. Again, this shows the commutation rules (3.17) are valid for all $n$ 's.

The next step is the derivation of the invariant $K(X, Y)$. In the commutative case the denominator would be $(X Y-Z)(X Y-1)$. Here it is not sufficient to consider an appropriate ordering. It turns out that the correct quantity that has to replace $W^{-1}$ in (2.39) (which can be written in a form similar to (2.32)) is not the natural inverse of some quantity $U$ as in the previous case, but rather the quantity:

$$
S=(X Y-1)^{-1}(X Y-Z)^{-1}+(Y X-1)^{-1}(Y X-1)^{-1}-\frac{(q-1)^{2}}{q(Z-1)^{2}}
$$

which (up to a trivial factor 2 ) goes over, when $q=1$, to the quantity that appears in the commutative case. Remarkably, $S$ is already invariant under the exchange of $X$ and $Y$ and is the same whether sandwiched between $X^{a}$ and $Y^{b}$ or between $Y^{b}$ and $X^{a}$ in the analogue of (2.39).

The idea is again to write $K\left(x_{n-1}, y_{n}\right)$ in terms of $w_{n}, w_{n}^{-1}$ and functions of $y_{n}$ only. The first step is to note that

$$
\left(x_{n-1} y_{n}-Z\right)\left(x_{n-1} y_{n}-1\right)^{-1}=
$$




$$
x_{n-1}\left(y_{n} x_{n-1}-Z\right)\left(y_{n} x_{n-1}-1\right)^{-1} x_{n-1}^{-1}=x_{n-1} w_{n} x_{n-1}^{-1}
$$

and from (3.17a)

$$
\left(x_{n-1} y_{n}-Z\right)\left(x_{n-1} y_{n}-1\right)^{-1}=q^{-1} w_{n}
$$

so the quantity in the l.h.s. can be expressed in terms of $w_{n}$. Next we rewrite $S$ in two different ways:

$$
\begin{aligned}
S= & \frac{q+1}{q(Z-1)^{2}}\left(q(X Y-Z)^{-1}-(X Y-1)^{-1}\right)= \\
& \frac{q+1}{q(Z-1)^{2}}\left((Y X-Z)^{-1}-q(Y X-1)^{-1}\right)
\end{aligned}
$$

Using $1=(Z-1)^{-1}((Y X-1)-(Y X-Z))$ we see that $S\left(x_{n-1}, y_{n}\right)$ can be written as a linear combination of $w_{n}, w_{n}^{-1}$ and constants. So the $h$ terms in $K\left(x_{n-1}, y_{n}\right)$ will give terms containing only $w_{n}, w_{n}^{-1}$ and functions of $y_{n}$. Similarly we write $X$ as $Y^{-1}(Z-$ $1)^{-1}(Z(Y X-1)-(Y X-Z))$. Multiplying this expression for $x_{n-1}$ on the right by the last expression for $S\left(x_{n-1}, y_{n}\right)$ we see that $x_{n-1} S\left(x_{n-1}, y_{n}\right) g\left(y_{n}\right)$ will also be expressed by $w_{n}, w_{n}^{-1}$ and functions of $y_{n}$. For $g\left(y_{n}\right) S\left(x_{n-1}, y_{n}\right) x_{n-1}$ we use the other expression of $S$ and a different rewriting of $X$. So far only the fact that $S$ can be expressed as a sum of pure denominators was used, the exact expression was not crucial. For the quantity $X^{2} S f\left(y_{n}\right)$ it becomes delicate. We have

$$
\begin{gathered}
x_{n-1} S\left(x_{n-1}, y_{n}\right)=\frac{q+1}{q(Z-1)^{3}} y_{n}^{-1}\left(Z\left(y_{n} x_{n-1}-1\right)-\right. \\
\left.\left(y_{n} x_{n-1}-Z\right)\right)\left(\left(y_{n} x_{n-1}-Z\right)^{-1}-q\left(y_{n} x_{n-1}-1\right)^{-1}\right)= \\
\frac{q+1}{q(Z-1)^{3}} y_{n}^{-1}\left(Z w_{n}^{-1}-1-q Z+q w_{n}\right)=\frac{q+1}{q(Z-1)^{3}}\left(q Z w_{n}^{-1}-1-q Z+w_{n}\right) y_{n}^{-1}
\end{gathered}
$$

where we have used (3.17) to commute $y_{n}$ and $w_{n}$. Because the $w_{n}$ independent part is exactly $-1-q Z$ we can regroup the terms between parentheses as $q Z\left(w_{n}^{-1}-1\right)+\left(w_{n}-1\right)$ and each term is a pure denominator. (A similar phenomenon helped us already in the previous case, when $\nu$ terms coming from commuting $y_{n}$ did combine to cancel each other). Next, multiplying again from the left by $x_{n-1}$ written in terms of $\left(y_{n} x_{n-1}-Z\right)$ and $\left(y_{n} x_{n-1}-1\right)$ we recover expressions of $w_{n}, w_{n}^{-1}$ and functions of $y_{n}$ only. That would not have been true had a different constant term been present in the right hand side of (3.22) and this is why we need the additive term in $S$. The same manipulation must be done, using the other expression for $S$ and $X$, for the $f\left(y_{n}\right) S\left(x_{n-1}, y_{n}\right) x_{n-1}$ term. It turns out that the very same cancellation happens and only $w_{n}, w_{n}^{-1}$ and functions of $y_{n}$ appear. Since we know how to commute $w_{n}$ with any function $j$ of $y_{n}$, through $j\left(y_{n}\right) w_{n}=w_{n} j\left(q y_{n}\right)$, we can finally write $K\left(x_{n-1}, y_{n}\right)$ as

$$
K\left(x_{n-1}, y_{n}\right)=w_{n} P\left(y_{n}\right)+Q\left(y_{n}\right)+R\left(y_{n}\right) w_{n}^{-1}
$$

where $P, Q$ and $R$ are complicated functions of $f, g$ and $h$. Again, everything done with $v_{n+1}$, moving from left to right has precisely the same effect as moving $w_{n}$ from right to left and conversely. So manipulating $K\left(x_{n+1}, y_{n}\right)$ in the opposite direction we get:

$$
K\left(x_{n+1}, y_{n}\right)=P\left(y_{n}\right) v_{n+1}+Q\left(y_{n}\right)+v_{n+1}^{-1} R\left(y_{n}\right)
$$


Again, if $v_{n+1} w_{n}=P\left(y_{n}\right)^{-1} R\left(y_{n}\right), K$ is conserved and the mapping is just (3.14a) with $F=P^{-1} R$. Since we started from the analogue of (2.39) it follows that the derivation of (3.14b) goes along the very same lines.

III) The last system we will consider is

$$
\begin{gathered}
\left(x_{n+1}+y_{n}\right)^{-1}+\left(x_{n-1}+y_{n}\right)^{-1}=F\left(y_{n}\right) \\
\left(x_{n+1}+y_{n+2}\right)^{-1}+\left(x_{n+1}+y_{n}\right)^{-1}=G\left(x_{n+1}\right)
\end{gathered}
$$

In the commutative (nonautonomous) case, this is one of the possible forms for the discrete equation which is the nonlinear contiguity of the Painlevé VI equation $[10,11]$.

Defining $w_{n}=\left(x_{n-1}+y_{n}\right)^{-1}$ and $v_{n+1}=\left(x_{n+1}+y_{n}\right)^{-1}$, the system (3.25) augmented by the definitions of $w_{n}$ and $v_{n+1}$ leads to:

$$
\begin{gathered}
y_{n}+x_{n-1}=w_{n}^{-1} \\
v_{n+1}+w_{n}=F\left(y_{n}\right) \\
x_{n+1}+y_{n}=v_{n+1}^{-1} \\
w_{n+2}+v_{n+1}=G\left(x_{n+1}\right)
\end{gathered}
$$

still following the sequence of variables (3.4). Since all equations are of the additive type, the commutation relations are just

$$
\begin{gathered}
w_{n} x_{n-1}=x_{n-1} w_{n}+\nu \\
y_{n} w_{n}=w_{n} y_{n}+\nu \\
v_{n+1} y_{n}=y_{n} v_{n+1}+\nu \\
x_{n+1} v_{n+1}=v_{n+1} x_{n+1}+\nu
\end{gathered}
$$

and from $(3.27 \mathrm{~d})$ one gets $w_{n+2} x_{n+1}=x_{n+1} w_{n+2}+\nu$, i.e. (3.27a) at $n+2$. Here the invariant $K$ is very similar to $(2.32)$ (or $(2.39)$ ) with $(X+Y)^{-2}$ replacing $(X+Y)^{-1}$ everywhere. As in the first case of this section we will replace $X$ in the equivalent of (2.32) by $(X+Y)-Y$. But contrary to that cases where the known commutation rules did not apply directly to the "sandwiched" object $U^{-1}$ or $S$ and manipulations were needed just to reconstruct an object we knew how to commute, in the present case, the object are just $w_{n}^{2}\left(\right.$ or $v_{n+1}^{2}$ ) and the commutation is immediate. Therefore it is obvious that $K\left(x_{n-1}, y_{n}\right)$ can be written as:

$$
K\left(x_{n-1}, y_{n}\right)=P\left(y_{n}\right) w_{n}^{2}+Q\left(y_{n}\right) w_{n}+R\left(y_{n}\right)
$$

Powers of $w_{n}$ less than 2 come, both from the $w_{n}^{-1}$ in the expression of $x_{n-1}=w_{n}^{-1}-y_{n}$, (coming with the $f$ and $g$ terms in the analogue of (2.32)), and from commutations of $w_{n}$ with functions of $y_{n}$, to bring everything on the side we want. Now we use again the argument that anything done with $w_{n}$ from left to right is identical to the same operation $v_{n+1}$ from right to left. So we have:

$$
K\left(x_{n+1}, y_{n}\right)=v_{n+1}^{2} P\left(y_{n}\right)+v_{n+1} Q\left(y_{n}\right)+R\left(y_{n}\right)
$$


with exactly the same $P, Q, R$. Thus using a consequence of $(3.27 \mathrm{~b})$, analogous to (2.7):

$$
\begin{gathered}
K\left(x_{n-1}, y_{n}\right)-R=\left(P w_{n}+Q\right) w_{n}=\left(w_{n} P+\nu P^{\prime}+Q\right) w_{n}= \\
\left(w_{n}+\left(\nu P^{\prime}+Q\right) P^{-1}\right) P w_{n}
\end{gathered}
$$

where the prime denotes the derivative with respect to $y_{n}$ and we have dropped the explicit $y_{n}$ dependence. Using a similar consequence of (3.24c) we get

$$
\begin{gathered}
K\left(x_{n+1}, y_{n}\right)-R=v_{n+1}\left(v_{n+1} P+Q\right)=v_{n+1}\left(P v_{n+1}+\nu P^{\prime}+Q\right)= \\
v_{n+1} P\left(v_{n+1}+\left(\nu P^{\prime}+Q\right) P^{-1}\right)
\end{gathered}
$$

These two expressions coincide if we take $w_{n}+v_{n+1}+\left(\nu P^{\prime}+Q\right) P^{-1}=0$, i.e. $F=-\left(\nu P^{\prime}+\right.$ Q) $P^{-1}$ in (3.25a). Since $K$ is also quadratic in $Y$, (3.25b) follows from the same arguments.

\section{On the integrability of mappings with non-commuting variables}

In the previous sections we have examined a family of second-order mappings of QRT type and have shown that it was possible to introduce consistent commutation rules and ensure the conservation of an invariant. But what does this means as far as integrability is concerned? In the classical, commutative, case the existence of the invariant has as consequence that the mapping is solvable in terms of elliptic functions. This solution exists at the commutative limit of the mapping presented here, but the solution in the general non-commutative case is not known.

It would thus be interesting to examine the mappings obtained here with other methods which have proven useful for the investigation of integrability in the commutative case. As such we choose singularity confinement [12] and algebraic entropy [13] (which is related to the degree growth of the iterates). We choose a simple mapping belonging to the first case $(2.2,2.8)$ treated here:

$$
x_{n+1}+x_{n-1}=a+b_{n} x_{n}^{-1}-c_{n}\left(a-x_{n}\right)^{-1}
$$

where $x_{n}, x_{n \pm 1}$ obey a Heisenberg commutation relation, $a, b_{n}, c_{n}$ are $c$-numbers and moreover $a$ is taken to be $n$-idependent. We shall start with initial conditions $x_{n-1}=p$, $x_{n}=q$, with $[p, q]=k$, and investigate the singularity properties of the mapping when $q$ happens to go through a non-invertible value. Iterating we find: $x_{n+1}=u q^{-1}$ with $u=$ $-p q+a q+b_{n}-c_{n}(a-q)^{-1} q$, and $x_{n+2}=a-q v$ with $v=1-b_{n+1} u^{-1}+c_{n+1}(a q-u)^{-1}$. We see readily that when $q$ becomes non-invertible, $x_{n+1}$ would be singular, and so would $(a-$ $\left.x_{n+2}\right)^{-1}=v^{-1} q^{-1}$. Then $x_{n+3}$ contains several singular contributions. In fact we can write $x_{n+3}=a+R q^{-1}$ where $R=b_{n+2} u^{-1}-c_{n+2} v^{-1}$ is regular when $q$ becomes non-invertible. Moreover a detailed calculation shows that, under the condition $b_{n+1}-b_{n}=c_{n+2}-c_{n+1}$, $R$ can be written $R=S q$ with $S$ regular when $q$ non-invertible, so $x_{n+3}$ is well-defined. This cancellation of the singular factor $q^{-1}$ is tantamount to singularity confinement in the commutative case. The same holds true when one examines the singularity where $a-q$ becomes noninvertible. The cancellation then requires $b_{n+2}-b_{n+1}=c_{n+1}-c_{n}$. So for cancellation in both cases we need

$$
c_{n+2}-c_{n+1}-c_{n}+c_{n-1}=0
$$


and

$$
b_{n}=c_{n-1}+c s t
$$

We have thus $c_{n}=\alpha n+\beta+\gamma(-1)^{n}$ (and $b_{n}=\alpha n+\delta-\gamma(-1)^{n}$ ). (This even-odd dependence is exactly what would reduce (4.1) to the system (2.2-2.8) in the autonomous case $\alpha=0$, by calling $y$ 's the $x$ 's at even indices). Thus the singularity that develops when $q$ or $a-q$ are non-invertible is confined. Moreover as in the commutative case this cancellation of the denominator is sufficient in order to curb the degree growth of (4.1) from exponential to polynomial. Thus the mapping has zero algebraic entropy. As a matter of fact the deautonominized symmetric form of (4.1) i.e. $\gamma=0$, was examined in [3], where we have presented its Lax pair in the case where $x_{n}, x_{n \pm 1}$ do not commute but rather obey a Heisenberg commutation rule. We have shown there that with:

$$
\begin{gathered}
L_{n}=\left(\begin{array}{cccc}
\lambda_{1} & x_{n} & 1 & 0 \\
0 & \lambda_{2} & c-x_{n-1} & 1 \\
h & 0 & \lambda_{3} & c-x_{n} \\
h x_{n-1} & h & 0 & \lambda_{4}
\end{array}\right) \\
M_{n}=\left(\begin{array}{cccc}
\left(\lambda_{1}-\lambda_{2}\right) x_{n}^{-1} & 1 & 0 & 0 \\
0 & 0 & 1 & 0 \\
0 & 0 & \left(\lambda_{3}-\lambda_{4}\right)\left(c-x_{n}\right)^{-1} & 1 \\
h & 0 & 0 & 0
\end{array}\right)
\end{gathered}
$$

where $\lambda_{1}=$ cnst., $\lambda_{3}=$ cnst., $\lambda_{2}=\frac{n-1}{2}$ and $\lambda_{4}=\frac{n}{2}$, the compatibility condition $h \frac{d M_{n}}{d h}=$ $L_{n+1} M_{n}-M_{n} L_{n}$ leads to

$$
x_{n+1}+x_{n-1}=c+\left(\lambda_{1}-\lambda_{2}\right) x_{n}^{-1}-\left(\lambda_{3}-\lambda_{4}\right)\left(c-x_{n}\right)^{-1}
$$

(note that equation 26 of [3] contained a small sign mistake). Eq. (4.3) is the extension of the discrete Painlevé II equation to the case of non-commuting variables. Thus the mapping (4.1) does satisfy several integrability criteria, and it is reasonable to surmise that it is indeed integrable. While such a detailed analysis is not in principle impossible (at least at the singularity confinement and algebraic entropy level) for the remaining cases treated in this paper, it is exceeedingly lengthy and will not be attempted here. We expect that, in parallel to the commuting case, the mappings examined here will satisfy the two basic integrability requirements.

Several questions remain open at this point. Once a Lax pair for non-commuting variables is obtained, how does one proceed to the actual integration of the mapping? As a matter of fact this question has been barely touched upon even in the commuting case. Restricting ourselves to the autonomous case, and assuming that the evidence on the integrability of the mapping is convincing, one can still wonder about the integrability of the correspondence defined by the conservation of the invariant $K$. In the commutative case it was shown in [14] that the growth of the number of images of a given point is linear in the number of iterations rather than exponential. From that low growth the integrability of the correspondence was assumed, long before its explicit integration in the general asymmetric case $[7,15]$. However adapting this proof to the non-commuting case does not seem possible. Indeed the number of solutions of an equation of degree 
two in a noncommutative variable may well be larger than two. This does not even take into consideration the possibility of zero divisors in the, so far indeterminate, space of objects we are considering. (In the field of quaternions, for instance, the equation $(X-i)(X-j)(X-k)-(X-k)(X-i)(X-j)=0$ is not an identity, is of second degree in $X$ and has at least four solutions, namely $i, j, k$ and 0$)$. Since there may be more than two solutions to the equation $K\left(X, y_{n}\right)=K\left(x_{n-1}, y_{n}\right)$ the fact that the two known solutions form a sub-tree of linear, rather than exponential growth, gives no indication on the growth of the full tree of all solutions. Thus the question of the integrability of the correspondence defined by the conservation of $K$ remains unanswered. However, even if it turned out to be nonintegrable, this would not be in contradiction with the (surmised) integrability of the mappings we derived here. It would just mean that the correspondence, retaining only the value of $K$, does not capture enough of the information present in the mapping to be integrable itself.

\section{Acknowledgments}

K.M. Tamizhmani acknowledges invitations from both Centre des Physique Théorique and Centre des Mathématiques of Ecole Polytechnique. T. Tamizhmani acknowledges an invitation from the Groupe de Modélisation Physique et Interfaces Biologie of Paris VII University. A. Ramani is grateful to J. Sauloy for interesting discussions.

\section{References}

[1] F.W. Nijhoff and H.W. Capel, Phys. Lett. A, 1992, V.163, 49

[2] G.R.W. Quispel and F.W. Nijhoff, Phys. Lett. A, 1992, V.161, 419.

[3] B. Grammaticos, A. Ramani, V. Papageorgiou, F. Nijhoff, J. Phys. A, 1992, V. 25, 6419.

[4] S.P. Novikov, Fuct. Anal. Appl., 1991, V. 24, 296.

[5] B. Grammaticos and A. Ramani, Reg. and Chaot. Dyn., 2000, V. 5, 53.

[6] G.R.W. Quispel, J.A.G. Roberts and C.J. Thompson, Physica D, 1989, V. 34, 183.

[7] A. Ramani, A. S. Carstea, B. Grammatics and Y. Ohta, Physica A, 2002, V. 305, 437.

[8] B. Grammaticos, Y. Ohta, A. Ramani and H. Sakai, J. Phys. A, 1998, V. 31, 3545.

[9] B. Grammaticos and A. Ramani, Phys. Lett. A, 1999, V. 257, 288.

[10] F.W. Nijhoff, A. Ramani, B. Grammaticos and Y. Ohta, Stud. Appl. Math., 2000, V. 106, 261.

[11] R. Conte and M. Musette, J. Phys. A, 2001, V. 34, 10507.

[12] B. Grammaticos, A. Ramani and V. Papageorgiou, Phys. Rev. Lett., 1991, V. 67, 1825.

[13] J. Hietarinta and C. Viallet, Phys. Rev. Lett., 1998, V. 81, 325.

[14] B. Grammaticos, F. Nijhoff and A. Ramani, Discrete Painlevé equations, in: The Painlevé Property: One Century Later (ed. R. Conte), CRM Series in Mathematical Physics, pp 413516, Springer-Verlag, New-York (1999).

[15] A. Iatrou and J.A.G. Roberts, J. Phys. A, 2001, V. 34, 6617. 